\title{
Periodic Travelling Wave Solutions of Discrete Nonlinear Schrödinger Equations
}

\author{
Dirk Hennig \\ Erich-Steinfurth-Str. 10/11, 16227 Eberswalde, Germany \\ Correspondence should be addressed to Dirk Hennig; hennigd@physik.hu-berlin.de
}

Received 29 August 2017; Revised 24 October 2017; Accepted 7 November 2017; Published 26 November 2017

Academic Editor: Erik Van Vleck

Copyright (C) 2017 Dirk Hennig. This is an open access article distributed under the Creative Commons Attribution License, which permits unrestricted use, distribution, and reproduction in any medium, provided the original work is properly cited.

\begin{abstract}
The existence of nonzero periodic travelling wave solutions for a general discrete nonlinear Schrödinger equation (DNLS) on onedimensional lattices is proved. The DNLS features a general nonlinear term and variable range of interactions going beyond the usual nearest-neighbour interaction. The problem of the existence of travelling wave solutions is converted into a fixed point problem for an operator on some appropriate function space which is solved by means of Schauder's Fixed Point Theorem.
\end{abstract}

\section{Introduction}

Coherent structures arising in the form of travelling waves, solitons, and breathers in systems of coupled oscillators have attracted considerable interest not least due to the important role they play for applications in physics, biology, and chemistry (for reviews see [1-3]). In this context a variety of nonlinear lattice systems has been studied including Fermi-Pasta-Ulam systems, discrete nonlinear Klein-Gordon systems, phase oscillator lattices, Josephson junction systems, reaction-diffusion systems, and the discrete nonlinear Schrödinger equation. Some exact results concerning the existence, stability, and uniqueness of coherent structures in the above-mentioned systems have been obtained; see, for example, [4-27].

In particular with regard to the existence of periodic travelling waves (TWs) in nonlinear lattice systems various methods have been used. For instance, the existence of small amplitude waves in nonlinear discrete Klein-Gordon systems was proved with the usage of spatial dynamics and centre manifold reduction [15, 21]. For the Frenkel-Kontorova model the existence of TWs was shown by means of fixed point methods in [16]. Utilising a modified Lyapunov-Schmidt technique, existence of periodic TWs in Newton's cradle was proved in [25]. Systems with nonlocal interactions were considered in $[19,24,28]$.
In [24] existence and bifurcation results for periodic TWs of a general infinite DNLS system as given in (1) in the next section were derived using variational methods. With the current study we present a (less involved) proof of the existence of periodic TWs for the same system but on finite lattices. To obtain our existence result, some appropriate function space is introduced on which the original problem is formulated as a fixed point problem for a corresponding operator. By exploiting Schauder's Fixed Point Theorem the existence of periodic TWs is established. The main advantage of considering finite lattices is that for periodic TW solutions the associated conserved norm (power), given in terms of a sum over the squares of the amplitudes at the lattice sites, is of finite value. Hereby the coercivity of the power can be used to define suitable subsets in function space in order to apply Schauder's Fixed Point Theorem. Nevertheless, imposing periodic boundary conditions an infinite lattice is constructed. The presented method can readily be applied to prove the existence of periodic TWs not only in general DNLS systems of higher dimensions but also in various other nonlinear lattice systems.

\section{General DNLS Systems}

In the current study we are interested in the existence of periodic TW solutions of the following general discrete 
nonlinear Schrödinger equation on finite one-dimensional lattices:

$$
i \frac{d \psi_{n}}{d t}=\sum_{j=1}^{N_{c}} \kappa_{j}\left[\psi_{n+j}-2 \psi_{n}+\psi_{n-j}\right]+F\left(\left|\psi_{n}\right|^{2}\right) \psi_{n},
$$

$$
1 \leq n \leq N
$$

with $\psi_{n} \in \mathbb{C}$.

The solutions satisfy periodicity conditions:

$$
\psi_{N+m}(t)=\psi_{m}(t) \text {, }
$$

for $m \in \mathbb{Z}$; namely, we consider the DNLS on rings. Moreover, by means of the periodic boundary conditions an infinite one-dimensional lattice can be obtained. Each unit interacts with its $N_{c}$ neighbouring oscillators to the left and right, respectively. $N_{c}=1, \ldots,[(N-1) / 2]$ determines the interaction radius, which ranges from nearest-neighbour interaction obtained for $N_{c}=1$ to global coupling when $N_{c}=(N-1) / 2$ and $N$ is odd.

Assume the following condition on $F\left(\left|\psi_{n}\right|^{2}\right)$ holds.

(A) $F \in C\left(\mathbb{R}_{+}, \mathbb{R}\right)$ for $\mathbb{R}_{+}=[0, \infty), F(0)=0$. There are constants $a>0, b>0$ such that

$$
|F(x)|<a\left(1+x^{b}\right)
$$

for any $x \geq 0$.

The standard DNLS, arising for $F\left(\left|\psi_{n}\right|^{2}\right)=\left|\psi_{n}\right|^{2}$ and $\kappa_{1} \neq 0, \kappa_{j \neq 1}=0$ in (1), is known to support periodic travelling wave solutions (see, e.g., [29]). As mentioned above the existence of periodic TWs in system (1) on the lattice $\mathbb{Z}$ was given in [24] using variational methods. Here we present a proof of the existence of periodic travelling wave solutions of (1) on finite lattices. Nevertheless, by imposing periodic boundary conditions an infinite lattice is constructed. For the existence statement we introduce some appropriate function space on which the original problem is converted into a fixed point problem for a corresponding operator. By means of Schauder's Fixed Point Theorem the existence of periodic TWs is established.

System (1) possesses two conserved quantities: the energy

$$
\begin{aligned}
\mathscr{H} & =\sum_{n=1}^{N}\left[G\left(\left|\psi_{n}\right|^{2}\right)-\sum_{m \neq n} \kappa_{m}\left|\psi_{m}-\psi_{n}\right|^{2}\right], \\
G(x) & =\int_{0}^{x} f(x) d x,
\end{aligned}
$$

and the power

$$
\mathscr{P}=\sum_{n=1}^{N}\left|\psi_{n}\right|^{2}
$$

We consider travelling wave solutions of the form

$$
\psi_{n}(t)=\Psi(k n-\omega t),
$$

with a $2 \pi$-periodic function $\Psi(u), u=k n-\omega t$, where $k \in$ $(-\pi, \pi)$ and $\omega \in \mathbb{R} \backslash\{0\}$ are the wave parameters.
In order for a travelling wave solution to satisfy the periodicity conditions in (2) we adopt the lattice size accordingly. This means that for a given wavenumber $|k|=\pi q$ with rational $q=r / s$ and two relatively prime integers $r, s \in$ $\mathbb{Z}_{+} \backslash\{0\}, r<s$, the number of sites of the lattice, $N$, is supposed to be an appropriate multiple of the minimal spatial period of the associated periodic travelling wave, determined by $L=s / r$, so that the periodicity conditions in (2) are fulfilled.

\section{Statement of the Existence Problem}

Regarding the existence of periodic travelling wave solutions we state the following.

Theorem 1. Let $(\mathbf{A})$ hold. Then for any rational number $q \in$ $\mathbb{Q} \cap(0,1)$ there exists nonzero periodic travelling wave solution $\psi_{n}(t)=\Psi(k n-\omega t) \equiv \Psi(u)$ of $(1)$ with $\Psi \in C^{1}(\mathbb{R}, \mathbb{C})$, such that

$$
\Psi(u+2 \pi)=\Psi(u), \quad \forall u \in \mathbb{R}
$$

provided that

$$
|\omega| \geq \mathscr{R}\left(1+p \frac{a\left(1+\mathscr{P}^{b}\right)}{\mathscr{R}(1+q)+4 \bar{\kappa}+a\left(1+\mathscr{P}^{b}\right)}\right),
$$

where

$$
\begin{aligned}
& \bar{\kappa}=\sum_{j=1}^{N_{c}} \kappa_{j}, \\
& p=\tilde{q}+\frac{1}{1-q}, \\
& \tilde{q}= \begin{cases}\frac{1}{q} & \text { for } 0<q<\frac{1}{2} \\
\frac{1}{1-q} & \text { for } \frac{1}{2} \leq q<1,\end{cases}
\end{aligned}
$$

and $\mathscr{R}$ determines the range $[-\mathscr{R}, \mathscr{R}]$ of the function $g \in C(\mathbb{R} \mid$ $\{0\}, \mathbb{R})$ given by

$$
g(x)=\frac{2}{x} \sum_{j=1}^{N_{c}} \kappa_{j} \sin ^{2}(j x)
$$

In the following we reformulate the original problem as a fixed point problem in a Banach space in a similar vein to the approach in [16].

\section{Proof of the Existence Theorem}

To prove the assertions of the theorem we utilise Schauder's Fixed Point Theorem (see, e.g., in [30]): let $M$ be a closed convex subset of a Banach space $X$. Suppose $T: M \rightarrow M$ is continuous mapping such that $T(M)$ is a relatively compact subset of $M$. Then $T$ has a fixed point. 
Proof. Travelling wave solutions $\Psi$ satisfy the advance-delay equation

$$
-i \omega \Psi^{\prime}(u)=\sum_{j=1}^{N_{c}} \kappa_{j} \Delta_{j} \Psi(u)+F\left(|\Psi(u)|^{2}\right) \Psi(u),
$$

where $\Delta_{j} \Psi(u)=\Psi(u+j)-2 \Psi(u)+\Psi(u-j)$ and $\Psi(u+$ $2 \pi)=\Psi(u), \forall u \in \mathbb{R}$, so that according to the Bloch-Floquet Theorem a solution must be of the form

$$
\Psi(u)=\exp (i q u) \Phi(u)
$$

where $q \in \mathbb{Q} \cap(0,1)$ and

$$
\Phi(u+2 \pi)=\Phi(u), \quad \forall u \in \mathbb{R} .
$$

Substituting (14) into (13) one obtains

$$
\begin{aligned}
-i \omega \Phi^{\prime}(u)+\omega q \Phi(u)= & \sum_{j=1}^{N_{c}} \kappa_{j} \widetilde{\Delta}_{j} \Phi(u) \\
& +F\left(|\Phi(u)|^{2}\right) \Phi(u),
\end{aligned}
$$

with

$$
\begin{aligned}
\widetilde{\Delta}_{j} \Phi(u)= & \Phi(u+j) \exp (i q j)-2 \Phi(u) \\
& +\Phi(u-j) \exp (-i q j)
\end{aligned}
$$

Thus, the task amounts to finding $2 \pi$-periodic functions $\Phi \in C^{1}(\mathbb{R}, \mathbb{C})$ satisfying $(16)$.

For the forthcoming discussion (16) is suitably rearranged as follows:

$$
\begin{aligned}
& -i \omega \Phi^{\prime}(u)+\omega q \Phi(u)-\sum_{j=1}^{N_{c}} \kappa_{j} \widetilde{\Delta}_{j} \Phi(u) \\
& =F\left(|\Phi(u)|^{2}\right) \Phi(u) .
\end{aligned}
$$

Note that terms nonlinear in $\Phi$ feature only on the r.h.s. of (18).

Let $q \in \mathbb{Q} \cap(0,1)$ be fixed. We identify $\mathbb{C}$ with $\mathbb{R}^{2}$. Denote by $X_{q}^{h}$ the real Banach spaces

$$
X_{q}^{h}=\left\{\Theta \in C_{2 \pi}^{h}(\mathbb{R}, \mathbb{C})\right\}, \quad h=0,1
$$

$$
\begin{aligned}
\left\|M_{q}^{-1}\right\|_{X_{q}^{0}, X_{q}^{1}} & \equiv\left\|M_{q}^{-1}\right\|=\sup _{0 \neq \Theta \in X_{q}^{0}} \frac{\left\|M_{q}^{-1} \Theta\right\|_{X_{q}^{1}}}{\|\Theta\|_{X_{q}^{0}}}=\sup _{0 \neq \Theta \in X_{q}^{0}} \frac{\left\|\sum_{l \in \mathbb{Z}}\left(1 / \nu_{l}\right) \Theta_{l} \exp (i l u)\right\|_{X_{q}^{1}}}{\|\Theta\|_{X_{q}^{0}}} \\
& =\sup _{0 \neq \Theta \in X_{q}^{0}} \frac{\left(\sup _{u \in[0,2 \pi]}\left|\sum_{l \in \mathbb{Z}}\left(1 / \nu_{l}\right) \Theta_{l} \exp (i l u)\right|+\sup _{u \in[0,2 \pi]}\left|\left(\sum_{l \in \mathbb{Z}}\left(1 / \nu_{l}\right) \Theta_{l} \exp (i l u)\right)^{\prime}\right|\right)}{\|\Theta\|_{X_{q}^{0}}} \\
& \leq \sup _{l \in \mathbb{Z}} \frac{1+|l|}{\left|\nu_{l}\right|} \sup _{0 \neq \Theta \in X_{q}^{0}} \frac{\sup _{u \in[0,2 \pi]}\left|\sum_{l \in \mathbb{Z}} \Theta_{l} \exp (i l u)\right|}{\|\Theta\|_{X_{q}^{0}}}=\sup _{l \in \mathbb{Z}} \frac{1+|l|}{\left|v_{l}\right|}
\end{aligned}
$$

respectively. $X_{q}^{1}$ is compactly embedded in $X_{q}^{0}\left(X_{q}^{1} \Subset X_{q}^{0}\right)$.

We decompose functions $\Theta \in X_{q}^{1}$ in a Fourier series

$$
\Theta(u)=\sum_{l \in \mathbb{Z}} \Theta_{l} \exp (i l u)
$$
$M_{q}: X_{q}^{1} \rightarrow X_{q}^{0}:$

$$
M_{q}(\Theta)=-i \omega \Theta^{\prime}(u)+\omega q \Theta(u)-\sum_{j=1}^{N_{c}} \kappa_{j} \widetilde{\Delta}_{j} \Theta(u) .
$$
upper bound for the norm of its inverse. $\exp (i l u)$ in $(21)$ results in

$$
M_{q} \exp (i l u)=v_{l}(q) \exp (i l u)
$$

where $X_{q}^{1}$ one derives:

Related to the l.h.s. of (18) we consider the linear mapping:

We demonstrate that this mapping is invertible and get an

Applying the operator $M_{q}$ to the Fourier elements

By the assumption (8) one has $\nu_{l}(q) \neq 0, \forall l \in \mathbb{Z}$, so that the mapping $M_{q}$ possesses an inverse obeying $M_{q}^{-1} \exp (i l u)=$ $\left(1 / \nu_{l}\right) \exp (i l u)$. For the bounded linear operator $M_{q}^{-1}: X_{q}^{0} \rightarrow$ 


$$
\begin{aligned}
& =\sup _{l \in \mathbb{Z}} \frac{1+|l|}{\left|(q+l)\left(\omega+(4 /(q+l)) \sum_{j=1}^{N_{c}} \kappa_{j} \sin ^{2}(((q+l) / 2) j)\right)\right|} \leq\left(\widetilde{q}+\frac{1}{1-q}\right) \frac{1}{|\omega|-\mathscr{R}} \\
& \leq \frac{(1+q) \mathscr{R}+4 \bar{\kappa}+a\left(1+\mathscr{P}^{b}\right)}{a\left(1+\mathscr{P}^{b}\right) \mathscr{R}},
\end{aligned}
$$

where $\widetilde{q}$ is given in (11).

For periodic travelling wave solutions $\Phi \in C_{2 \pi}^{1}(\mathbb{R}, \mathbb{C})$ one derives, using (3), (5), (8), and (16), the bounds

$$
\begin{array}{ll}
\max _{u \in[0,2 \pi]} & |\Phi(u)| \leq \mathscr{P}^{1 / 2}, \\
\max _{u \in(0,2 \pi)} & \left|\Phi^{\prime}(u)\right| \leq\left(q+\frac{4 \bar{\kappa}+a\left(1+\mathscr{P}^{b}\right)}{\mathscr{R}}\right) \mathscr{P}^{1 / 2} .
\end{array}
$$

We consider then the closed and convex subsets of $X_{q}^{0}$ and $X_{q}^{1}$ determined by

$$
\begin{aligned}
Y_{q}^{0} & =\left\{\Theta \in X_{q}^{0}:\|\Theta\|_{C_{2 \pi}^{0}} \leq \mathscr{P}^{1 / 2}\right\}, \\
Y_{q}^{1} & =\left\{\Theta \in X_{q}^{1}:\|\Theta\|_{C_{2 \pi}^{1}}\right. \\
& \left.\leq\left(1+q+\frac{4 \bar{\kappa}+a\left(1+\mathscr{P}^{b}\right)}{\mathscr{R}}\right) \mathscr{P}^{1 / 2}\right\},
\end{aligned}
$$

respectively. $Y_{q}^{1}$ is compactly embedded in $Y_{q}^{0}\left(Y_{q}^{1} \Subset Y_{q}^{0}\right)$.

Furthermore associated with the r.h.s. of (18) we introduce the nonlinear operator $N_{q}: Y_{q}^{0} \rightarrow Y_{q}^{0}$, as

$$
N_{q}(\Theta)=F\left(|\Theta|^{2}\right) \Theta \text {. }
$$

Clearly, the operator $N_{q}$ is uniformly continuous on $Y_{q}^{0}$. The range is contained in a bounded ball in $Y_{q}^{0}$, since

$$
\begin{aligned}
\left\|N_{q}(\Theta)\right\|_{Y_{q}^{0}} & =\left\|F\left(|\Theta|^{2}\right) \Theta\right\|_{C_{2 \pi}^{0}} \\
& =\max _{u \in[0,2 \pi]}\left|F\left(|\Theta(u)|^{2}\right) \Theta(u)\right| \\
& \leq a\left(1+\mathscr{P}^{b}\right) \mathscr{P}^{1 / 2} .
\end{aligned}
$$

Finally, we express problem (18) as a fixed point equation in terms of a mapping $Y_{q}^{0} \rightarrow Y_{q}^{1} \Subset Y_{q}^{0}$ :

$$
\Phi=M_{q}^{-1} \circ N_{q}(\Phi) \equiv T_{q}(\Phi) \text {. }
$$

We get

$$
\begin{aligned}
\left\|T_{q}(\Phi)\right\|_{Y_{q}^{1}} & =\left\|M_{q}^{-1}\left(N_{q}(\Phi)\right)\right\| \leq\left\|M_{q}^{-1}\right\|\left\|N_{q}(\Phi)\right\|_{Y_{q}^{0}} \\
& \leq\left(1+q+\frac{4 \bar{\kappa}+a\left(1+\mathscr{P}^{b}\right)}{\mathscr{R}}\right) \mathscr{P}^{1 / 2},
\end{aligned}
$$

verifying that indeed

$$
T_{q}\left(Y_{q}^{0}\right) \subseteq Y_{q}^{1}
$$

Hence $T_{q}$ maps bounded subsets $Y_{q}^{0}$ of $X_{q}^{0}$ into relatively compact subsets $Y_{q}^{1}$ of $Y_{q}^{0}$.

It remains to prove that $T_{q}$ is continuous on $Y_{q}^{0}$. As $N$ is uniformly continuous on $Y_{q}^{0}$, one has $\forall t \in[0,2 \pi]$ and $\forall \Phi_{1}, \Phi_{2} \in Y_{q}^{0}$ that for a fixed arbitrary $\epsilon>0$ there exists $\delta>0$ such that

$$
\begin{aligned}
\left\|N_{q}\left(\Phi_{1}\right)(t)-N_{q}\left(\Phi_{2}\right)(t)\right\|_{Y_{q}^{0}} \\
<\frac{a\left(1+\mathscr{P}^{b}\right) \mathscr{R}}{(1+q) \mathscr{R}+4 \bar{\kappa}+a\left(1+\mathscr{P}^{b}\right)} \epsilon
\end{aligned}
$$

if $\left\|\Phi_{1}-\Phi_{2}\right\|_{Y_{q}^{0}}<\delta$. Hence, for arbitrary $\Phi_{1}, \Phi_{2} \in Y_{q}^{0}$, we have

$$
\begin{gathered}
\left\|M_{q}^{-1}\left(N_{q}\left(\Phi_{1}\right)\right)-M_{q}^{-1}\left(N_{q}\left(\Phi_{2}\right)\right)\right\|_{Y_{q}^{1} \Subset Y_{q}^{0}} \\
\leq\left\|M_{q}^{-1}\right\|\left\|N_{q}\left(\Phi_{1}\right)-N_{q}\left(\Phi_{2}\right)\right\|_{Y_{q}^{0}}<\epsilon
\end{gathered}
$$

verifying that $T_{q}(\Phi)$ is continuous on $Y_{q}^{0}$. Schauder's Fixed Point Theorem implies then that the fixed point equation $\Phi=$ $T_{q}(\Phi)$ has at least one solution.

Furthermore, the spectrum of linear plane wave solutions (phonons) arising for zero nonlinear term, determined by the r.h.s. of system (18), forms a continuous band with values in the interval $[-\mathscr{R}, \mathscr{R}]$. However, since by hypothesis (8) the values of the frequency of oscillations $\omega$ lie outside the range of the linear (phonon) band, the corresponding orbits are anharmonic. This necessitates amplitude-depending tuning of the frequency so that the latter comes to lie outside of the phonon spectrum. Thus it must hold that $\left\|N_{q}(\Phi)\right\|_{Y_{q}^{0}}=$ $\left\|F\left(|\Phi|^{2}\right) \Phi\right\|_{C_{2 \pi}^{0}} \quad \not \equiv \quad 0$ which is fulfilled only if $\Phi \not \equiv 0$. That is, the fixed point equation (30) possesses only nonzero solutions and the proof is finished.

\section{Summary}

To summarise, we have proven the existence of nonzero periodic travelling wave solutions for a general DNLS (including as a special case the standard DNLS) on finite onedimensional lattices. To this end the existence problem has been reformulated as a fixed point problem for an operator on a function space which is solved with the help of Schauder's Fixed Point Theorem. Our method can be straightforwardly 
extended not only to treating the general DNLS on lattices of higher dimension but also to other types of nonlinear lattice systems such as nonlinear discrete Klein-Gordon systems.

\section{Conflicts of Interest}

The author declares that there are no conflicts of interest.

\section{References}

[1] O. M. Braun and Y. S. Kivshar, The Frenkel-Kontorova Model: Concepts, Methods, and Applications, Theoretical and Mathematical Physics, Springer, 2004.

[2] M. Remoissenet, Waves Called Solitons, Advanced Texts in Physics, Springer, Berlin, Germany, 1999.

[3] T. Dauxois and M. Peyrard, Physics of Solitons, Cambridge University Press, Cambridge, UK, 2006.

[4] B. Zinner, G. Harris, and W. Hudson, "Traveling wavefronts for the discrete Fisher's equation," Journal of Differential Equations, vol. 105, no. 1, pp. 46-62, 1993.

[5] N. Flytzanis, B. A. Malomed, and J. A. D. Wattis, "Analysis of stability of solitons in one-dimensional lattices," Physics Letters A, vol. 180, no. 1-2, pp. 107-112, 1993.

[6] G. Friesecke and J. A. Wattis, "Existence theorem for solitary waves on lattices," Communications in Mathematical Physics, vol. 161, no. 2, pp. 391-418, 1994.

[7] D. Smets and M. Willem, "Solitary waves with prescribed speed on infinite lattices," Journal of Functional Analysis, vol. 149, no. 1, pp. 266-275, 1997.

[8] S.-N. Chow, J. Mallet-Paret, and W. Shen, “Traveling waves in lattice dynamical systems," Journal of Differential Equations, vol. 149, no. 2, pp. 248-291, 1998.

[9] A. Pankov, Travelling Waves and Periodic Oscillations in FermiPasta-Ulam Lattices, Imperial College Press, London, UK, 2005.

[10] D. B. Duncan, J. C. Eilbeck, H. Feddersen, and J. A. D. Wattis, "Solitons on lattices," Physica D: Nonlinear Phenomena, vol. 68, no. 1, pp. 1-11, 1993.

[11] J. Mallet-Paret, "The global structure of traveling waves in spatially discrete dynamical systems," Journal of Dynamics and Differential Equations, vol. 11, no. 1, pp. 49-127, 1999.

[12] G. Friesecke and R. L. Pego, "Solitary waves on FPU lattices. I. Qualitative properties, renormalization and continuum limit," Nonlinearity, vol. 12, no. 6, pp. 1601-1627, 1999.

[13] A. A. Pankov and K. Pflüger, "Travelling waves in lattice dynamical systems," Mathematical Methods in the Applied Sciences, vol. 23, no. 14, pp. 1223-1235, 2000.

[14] A. Carpio, S. J. Chapman, S. Hastings, and J. . McLeod, "Wave solutions for a discrete reaction-diffusion equation," European Journal of Applied Mathematics, vol. 11, no. 4, pp. 399-412, 2000.

[15] G. Iooss and K. Kirchgässner, "Travelling waves in a chain of coupled nonlinear oscillators," Communications in Mathematical Physics, vol. 211, no. 2, pp. 439-464, 2000.

[16] R. Mirollo and N. Rosen, "Existence, uniqueness, and nonuniqueness of single-wave-form solutions to Josephson junction systems," SIAM Journal on Applied Mathematics, vol. 60, no. 5, pp. 1471-1501, 2000.

[17] P. W. Bates, X. Chen, and A. J. Chmaj, "Traveling waves of bistable dynamics on a lattice," SIAM Journal on Mathematical Analysis, vol. 35, no. 2, pp. 520-546, 2003.
[18] D. E. Pelinovsky and V. M. Rothos, "Bifurcations of travelling wave solutions in the discrete NLS equations," Physica D: Nonlinear Phenomena, vol. 202, no. 1-2, pp. 16-36, 2005.

[19] P. W. Bates and C. Zhang, "Traveling pulses for the KleinGordon equation on a lattice or continuum with long-range interaction," Discrete and Continuous Dynamical Systems Series A, vol. 16, no. 1, pp. 235-252, 2006.

[20] T. R. Melvin, A. R. Champneys, P. G. Kevrekidis, and J. Cuevas, "Travelling solitary waves in the discrete Schrödinger equation with saturable nonlinearity: existence, stability and dynamics," Physica D: Nonlinear Phenomena, vol. 237, no. 4, pp. 551-567, 2008.

[21] G. James and Y. Sire, "Center Manifold Theory in the Context of Infinite One-Dimensional Lattices," in The Fermi-Pasta-Ulam Problem, vol. 728 of Lecture Notes in Physics, pp. 207-238, 2008.

[22] X. Chen, J.-S. Guo, and C.-C. Wu, "Traveling waves in discrete periodic media for bistable dynamics," Archive for Rational Mechanics and Analysis, vol. 189, no. 2, pp. 189-236, 2008.

[23] J. Cuevas, N. I. Karachalios, and F. Palmero, "Energy thresholds for the existence of breather solutions and travelling waves on lattices," Applicable Analysis: An International Journal, vol. 89, no. 9, pp. 1351-1385, 2010.

[24] M. Fečkan and V. M. Rothos, "Travelling waves of discrete nonlinear Schrödinger equations with nonlocal interactions," Applicable Analysis: An International Journal, vol. 89, no. 9, pp. 1387-1411, 2010.

[25] G. James, "Nonlinear waves in Newton's cradle and the discrete p-Schrödinger equation," Mathematical Models and Methods in Applied Sciences, vol. 21, no. 11, pp. 2335-2377, 2011.

[26] M. Syafwan, H. Susanto, S. M. Cox, and B. A. Malomed, "Variational approximations for traveling solitons in a discrete nonlinear Schrödinger equation," Journal of Physics A: Mathematical and Theoretical, vol. 45, no. 7, Article ID 075207, 2012.

[27] J.-S. Guo and Y.-C. Lin, “Traveling wave solution for a lattice dynamical system with convolution type nonlinearity," Discrete and Continuous Dynamical Systems - Series A, vol. 32, no. 1, pp. 101-124, 2012.

[28] M. Fečkan, M. Pospíšil, V. M. Rothos, and H. Susanto, "Periodic Travelling Waves of Forced FPU Lattices," Journal of Dynamics and Differential Equations, vol. 25, no. 3, pp. 795-820, 2013.

[29] J. C. Eilbeck and M. Johansson, "The discrete nonlinear Schrödinger equation-20 years on," in Proceedings of the Third Conference on Localization and Energy Transfer in Nonlinear Systems, L. Vazquez, R. S. MacKay, and M.-P. Zorzano, Eds., pp. 44-67, San Lorenzo de El Escorial Madrid, Spain, June 2002.

[30] E. Zeidler, Nonlinear Functional Analysis and Its Applications I: Fixed Point Theorems, Nonlinear Functional Analysis \& Its Applications, Springer, New York, NY, USA, 1998. 


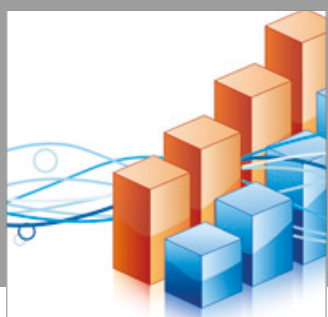

Advances in

Operations Research

vatersals

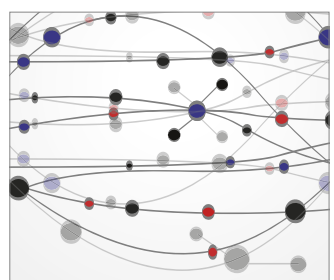

\section{The Scientific} World Journal
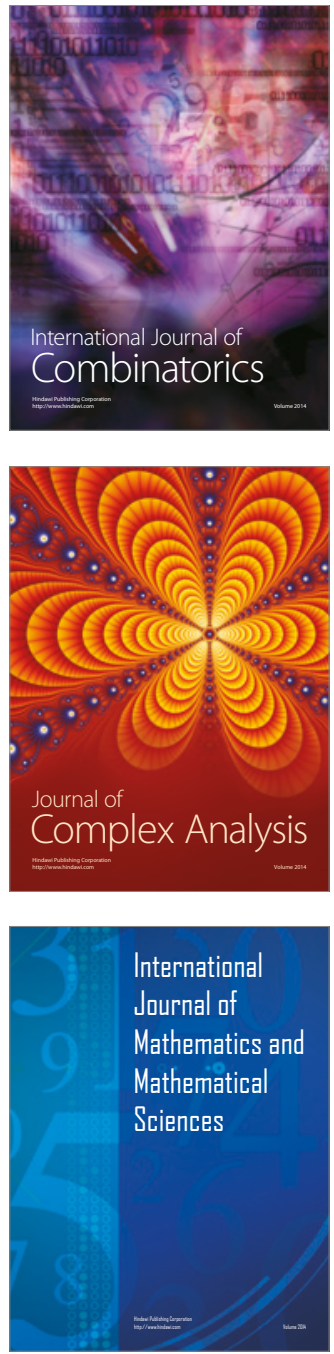
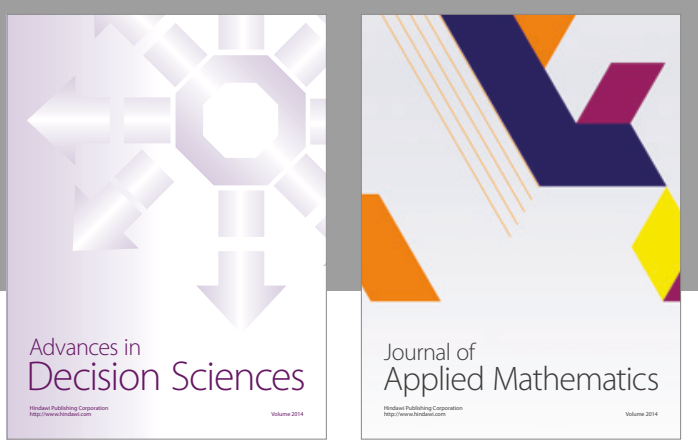

Algebra

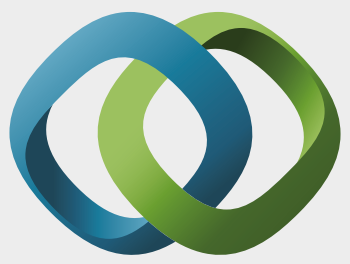

\section{Hindawi}

Submit your manuscripts at

https://www.hindawi.com
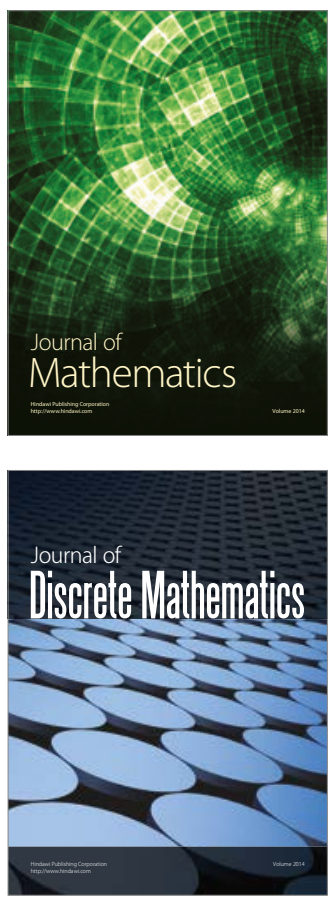

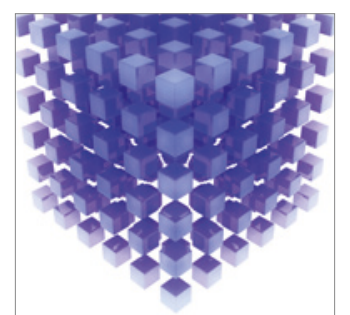

Mathematical Problems in Engineering
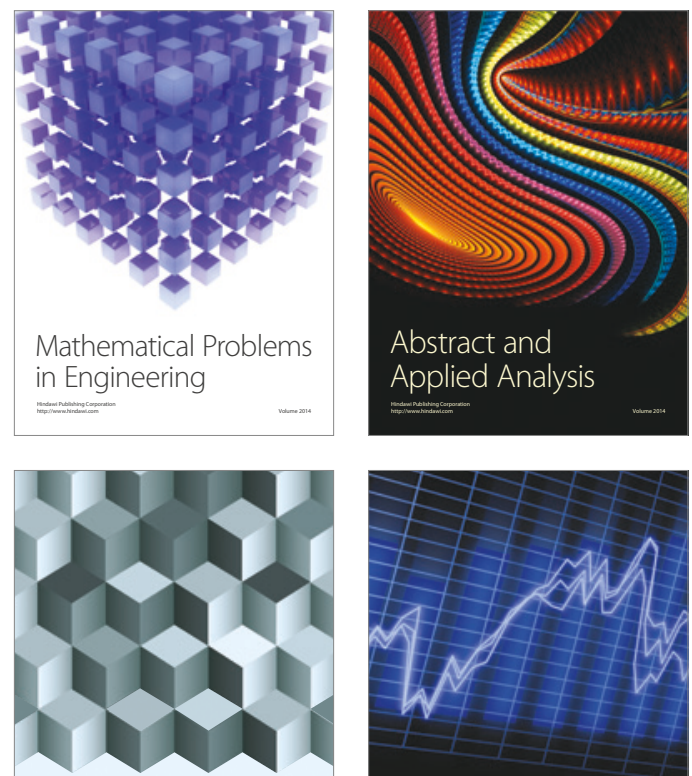

Journal of

Function Spaces

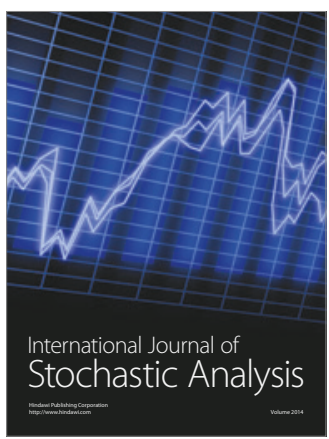

Probability and Statistics
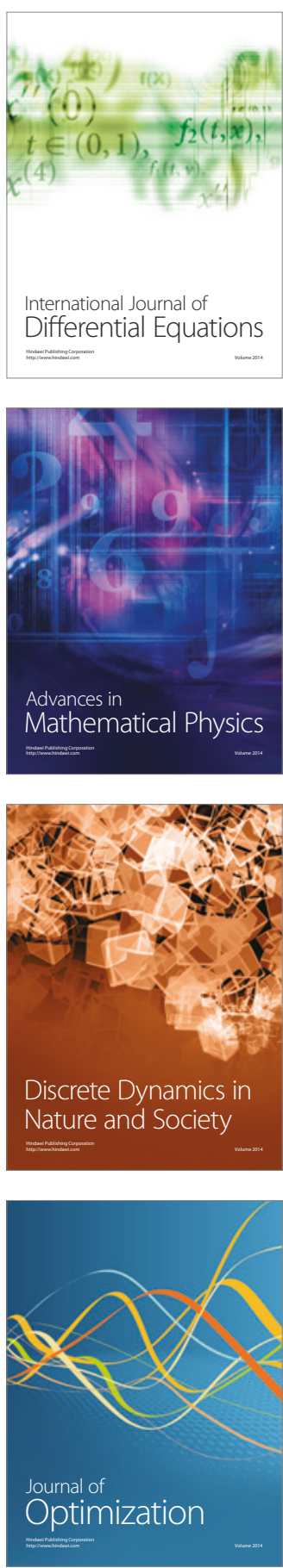